\title{
Efficacy of regorafenib in acute pulmonary carcinomatous lymphangitis as a manifestation of rectal cancer: A case report
}

\author{
NOBUMICHI TAKEUCHI ${ }^{1}$, KUMIKO KOIKE $^{1}$, SONOMI YOSHIDA $^{1}$ and MASAYUKI FUJIWARA ${ }^{2}$ \\ Departments of ${ }^{1}$ Medical Oncology and ${ }^{2}$ Clinical Pathology, Ina Central Hospital, Ina, Nagano 396-8555, Japan
}

Received May 31, 2019; Accepted September 26, 2019

DOI: $10.3892 / 01.2019 .11043$

\begin{abstract}
Recent therapeutic advancements have prolonged the survival duration of patients with metastatic or recurrent colorectal cancer even during salvage treatment. Although treatment with regorafenib and trifluridine/tipiracil combination has exhibited apparent survival benefits, clear and objective evidence of a response to these drugs is scarce. Herein, the present study reports the case of a patient with rectal cancer refractory to multiple surgical interventions and standard chemotherapy. Treatment with regorafenib resulted in immediate improvement of respiratory failure caused by pulmonary lymphangitic carcinomatosis. This improvement persisted for over 3 months and was confirmed by radiology. Our findings suggest that regorafenib can reduce peritumoral edema via its interaction with the vascular endothelial growth factor receptor. Thus, regorafenib functions as a multityrosine kinase inhibitor to alleviate symptoms of lymphangitic carcinomatosis despite the low potency of the drug.
\end{abstract}

\section{Introduction}

Regorafenib and trifluridine/tiperacil combination (TAS102) has shown survival benefit among patients with metastatic or recurrent colorectal cancer in cases where salvage treatment of fluoropyrimidine, irinotecan, oxaliplatin, anti-vascular endothelial growth factors (VEGFs), and anti-epidermal growth factor receptor therapy is required following failure of standard therapy. However, a clear and objective evidence of response of these drugs is rarely observed (1-4).

Herein, we report the case of a patient with rectal cancer refractory to multiple surgical interventions and standard chemotherapy, for whom administration of regorafenib treatment resulted in immediate improvement of respiratory failure caused by carcinomatous pulmonary lymphangitis in response

Correspondence to: Dr Nobumichi Takeuchi, Department of Medical Oncology, Ina Central Hospital, 1313-1 Koshirou Kubo, Ina, Nagano 396-8555, Japan

E-mail: ntakeuti@inahp.jp

Key words: regorafenib, rectal cancer, carcinomatous lymphangitis to regorafenib treatment. Written informed consent for publication was obtained from the next of kin.

\section{Case report}

A 65-year-old Japanese man underwent curative surgery for advanced rectal cancer with lymph node metastases at Ina Central Hospital without consecutive adjuvant treatment in April 2011. The tumor was diagnosed as Stage IIIB (T3N1bM0; AJCC-TNM 8th edition) by the pathological department of Ina Central Hospital. In October 2013, bilateral pulmonary metastases were detected by computed tomography without clinical symptom during routine follow-up and resected at Aizawa Hospital (Matsumoto city, Nagano, Japan). No definite histological differences were noted when compared with the specimen obtained during initial surgery. KRAS exon 2 codon $12 \mathrm{D}$ mutation was detected in the primary lesion and pulmonary metastases by Scorpion ARMS method (TheraScreen ${ }^{\circledR}$ : K-RAS Mutation kit). In March 2015, magnetic resonance imaging (MRI) revealed four brain metastases during the investigation of persistent headache, which were treated with stereotactic body radiotherapy (SBRT). Consecutively, followed by general chemotherapy with capecitabine $1,000 \mathrm{mg} / \mathrm{m}^{2}$ (twice daily for 2 weeks), $7.5 \mathrm{mg} / \mathrm{kg}$ bevacizumab and $130 \mathrm{mg} / \mathrm{m}^{2}$ oxaliplatine on day 1 repeated every 3 weeks until February 2016 when other brain metastases, multiple pulmonary metastases with pleural deposits, and multiple bone metastases were detected by MRI, computed tomography (CT) and positron emission tomography scans. After pleurodesis and SBRT for symptom palliation, general combination chemotherapy with $40 \mathrm{mg} / \mathrm{m}^{2}$ S-1 (a combination of tegafur, gimeracil and oteracil) twice daily for 2 weeks, and irinotecan $150 \mathrm{mg} / \mathrm{m}^{2}$ on day 1 repeated every 3 weeks was initiated in April 2016. After 3 weeks, the treatment was discontinued because of grade 3 general fatigue and poor appetite.

On June 23, 2016, the patient was admitted to Ina Central Hospital with dyspnea. Assessment involving chest $\mathrm{X}$-ray and CT, revealed cardiac tamponade (Fig. 1A and B). Pericardiocentesis detected $600 \mathrm{ml}$ of pericardial effusion, in which adenocarcinoma cells were detected. (Fig. 1C). The patient was discharged 4 days after pericardiocentesis; however, he was readmitted 2 days after discharge because of dyspnea. Unlike the previous admission, chest X-ray (Fig. 2A) and CT (Fig. 3) revealed an interstitial pattern in the upper lobe of the left lung without evident increase in pericardial effusion. 

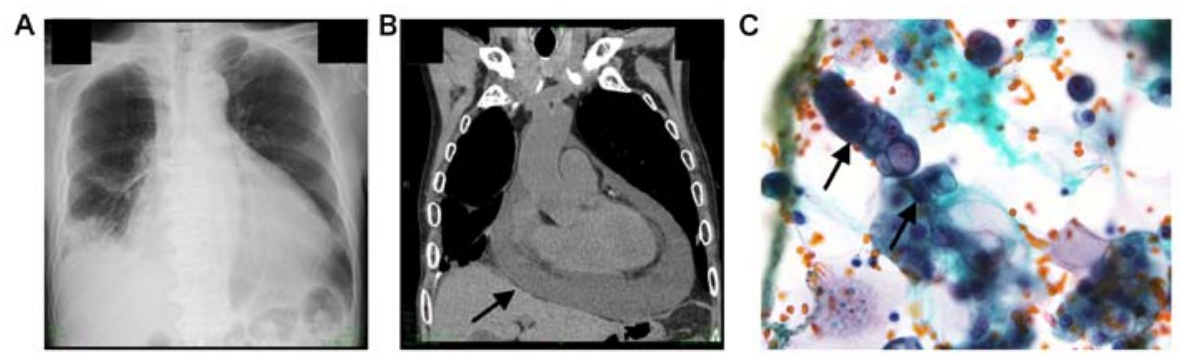

Figure 1. Radiographic and CT images of the chest obtained on June 23, 2016. Evident pericardial effusion and infiltrative shadow localized in the right lower lung from (A) chest X-ray and (B) CT. (C) Adenocarcinoma cells in pericardial effusion obtained by pericardiocentesis. CT, computed tomography.
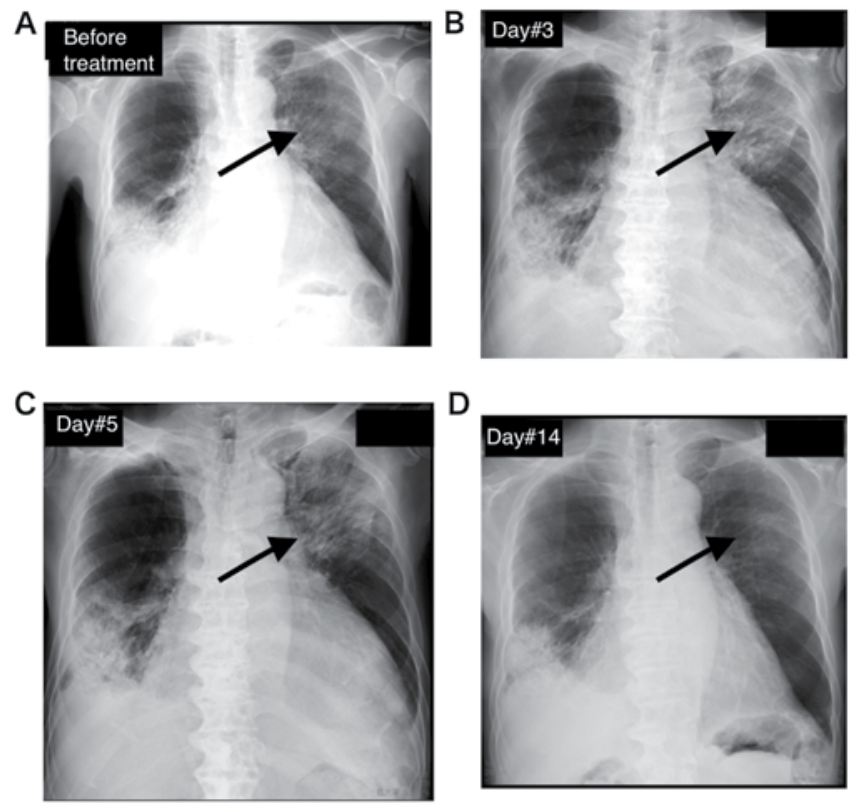

Figure 2. Radiographic image of chest during the early phase of regorafenib treatment. (A) Before treatment: A diffuse interstitial shadow in the left upper lobe (arrow) and localized infiltrative shadow in the right lower lobe. (B) Day 3. (C) Day 5. (D) Day 14. Quick and evident improvement of interstitial shadow was shown.

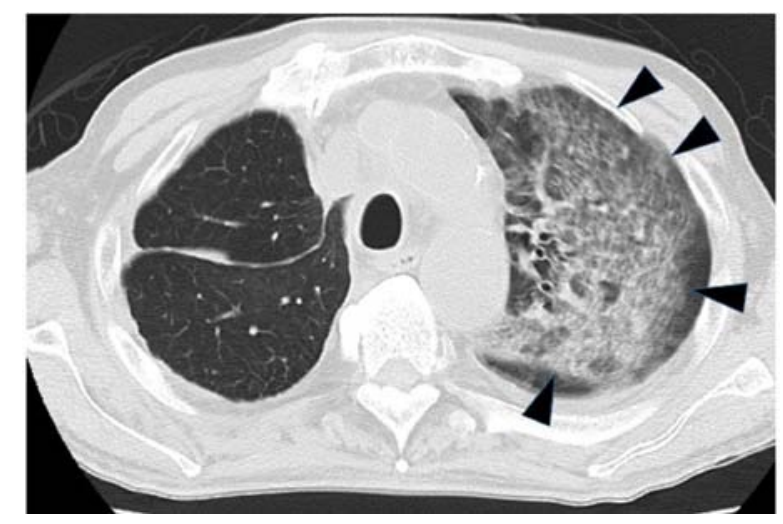

Figure 3. CT of chest on day 1 revealed ground-glass opacities in the left upper lobe (arrows). ground-glass opacities clearly improved. CT.

On the day after the readmission (day 1), once-daily oral administration of $120 \mathrm{mg}$ regorafenib was given for 21 consecutive days. Dyspnea was assessed by the Medical Research Council (MRC) Dyspnea Scale (5), following

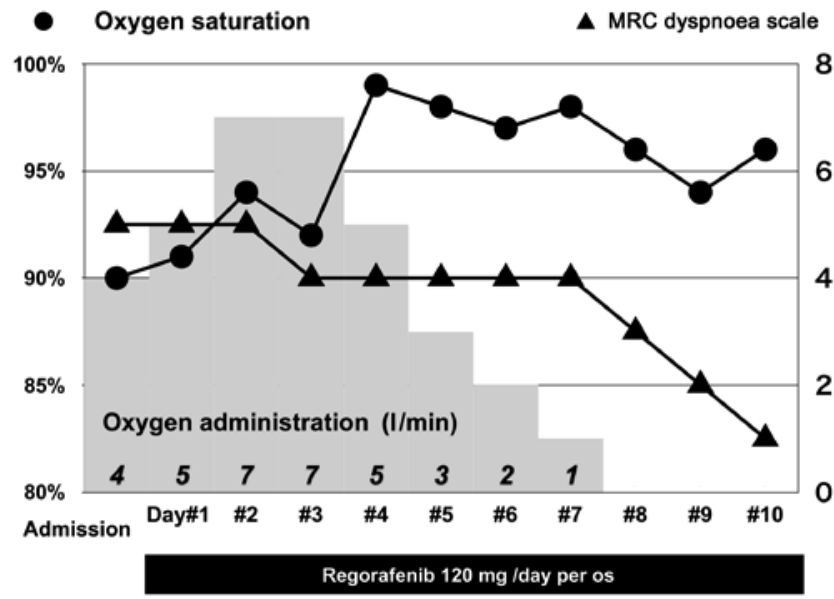

Figure 4. Clinical course after admission. Steady and quick improvement of respiratory failure by regorafenib. CT, computed tomography; MRC, medical research council.

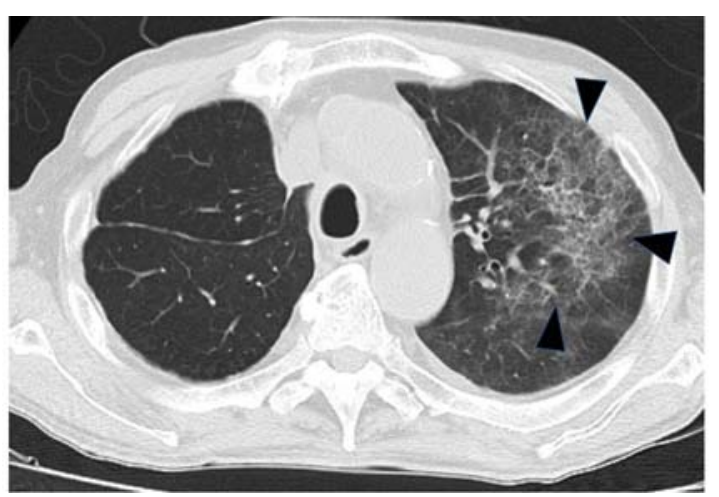

Figure 5. Computed tomography of chest on day 7 showed ground-glass opacities clearly improved (arrows).

hospitalization and oxygen saturation improved quickly and steadily following initiation of regorafenib. Oxygen administration was decreased gradually from day 4 until day 8 (Fig. 4). Chest X-ray revealed visible improvement of the interstitial pattern after treatment (Fig. 2B-D). Furthermore, CT on day 7 revealed clear improvement in the interstitial pattern (Fig. 5).

Regorafenib was orally administered for 21 days without dose adjustment. Hematological and non-hematological adverse effects did not occur until his discharge on day 22 . Regorafenib treatment as aforementioned was reinitiated at an outpatient clinic after a 7-day resting period (Fig. 6). The 
CEA

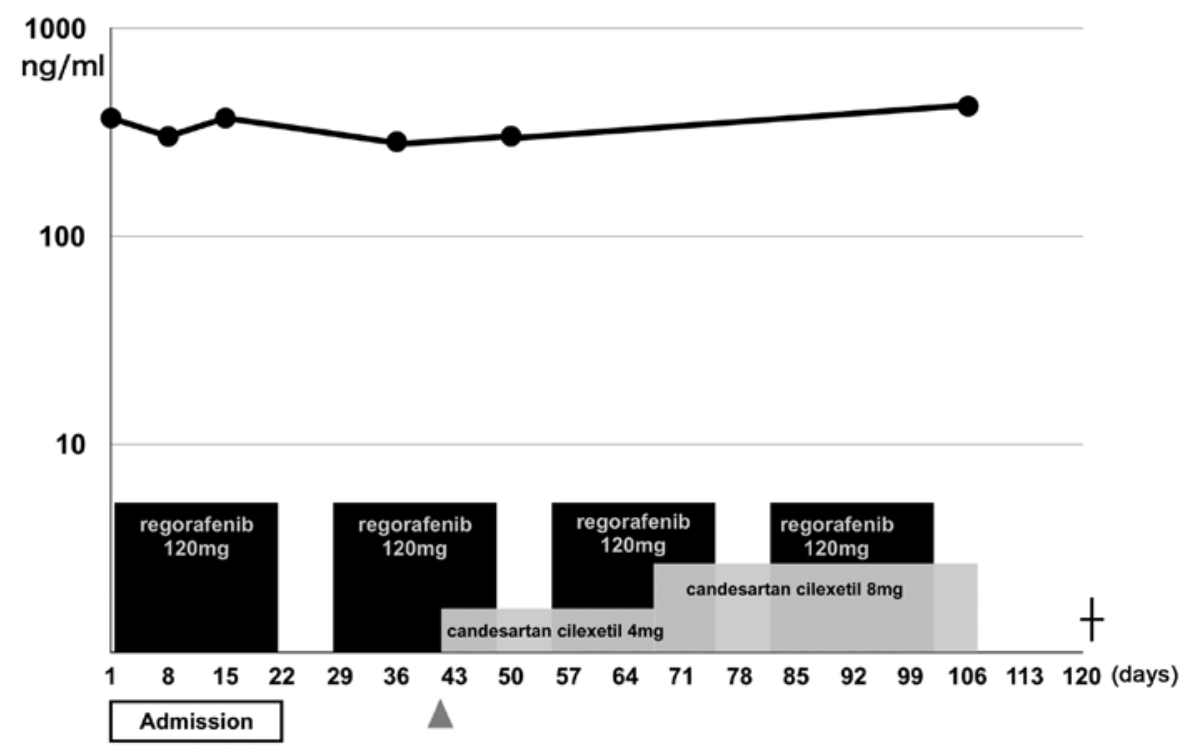

Figure 6. CEA level and the treatment course. No marked changes in elevated CEA levels were observed throughout the course of the treatment. Administration of candesartan cilexetile to treat grade 2 hypertension began during the second course of treatment $(\mathbf{\Lambda})$. CEA, carcinoembryonic antigen.

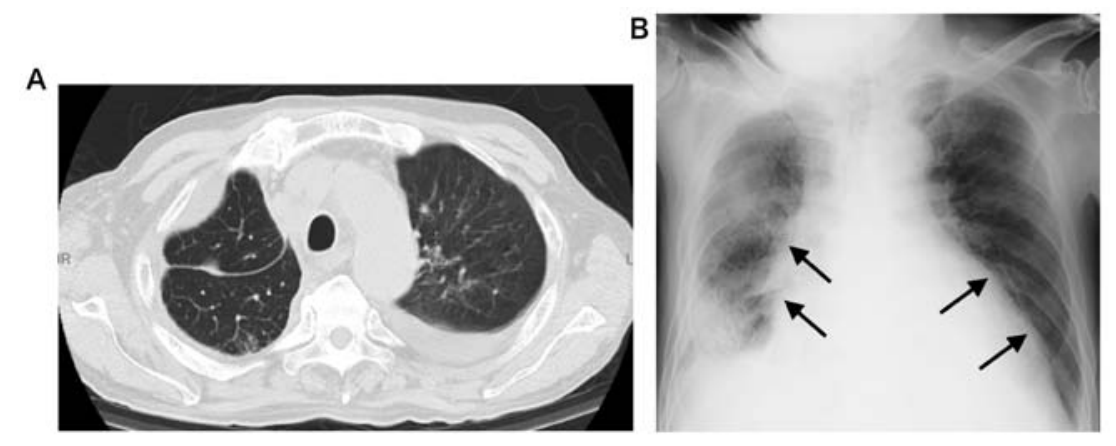

Figure 7. Chest imaging on day 109. (A) Radiographic imaging of chest. (B) CT of the chest. Marked pericardial effusion (arrows) without any pulmonary interstitial pattern was observed.

patient experienced grade 2 hypertension and required the administration of candesartan cilexetile, which was maintained by dose escalation during the second course of treatment $(\boldsymbol{\Lambda}$ in Fig. 6), and administration of regorafenib was withdrawn after they developed proteinuria (>1 g/dl) with hypoalbuminemia $(2.5 \mathrm{mg} / \mathrm{dl})$ and grade 4 renal function depletion (6) on day 18 of the fourth course of regorafenib treatment (103 days after initiation of treatment). The patient was readmitted with general fatigue and hypotension, which temporarily improved by pericardiocentesis, 6 days after discontinuation of regorafenib. Chest X-ray and CT did not indicate relapse of the interstitial pattern in the upper lobe of the left lung (Fig. 7). However, the patient died of cardiac tamponade on day 12 of admission, 121 days after initiation of regorafenib treatment.

Autopsy revealed cardiac tamponade caused by bloody pericardial effusion derived from disseminated nodules $(1,130 \mathrm{ml}$; Fig. 8), which was determined to be the direct cause of death. In addition, bilateral carcinomatous pleuritis with direct invasion of the attached lung was observed. Microscopic examination (magnifications, $\mathrm{x} 100$ or $\mathrm{x} 200$ )

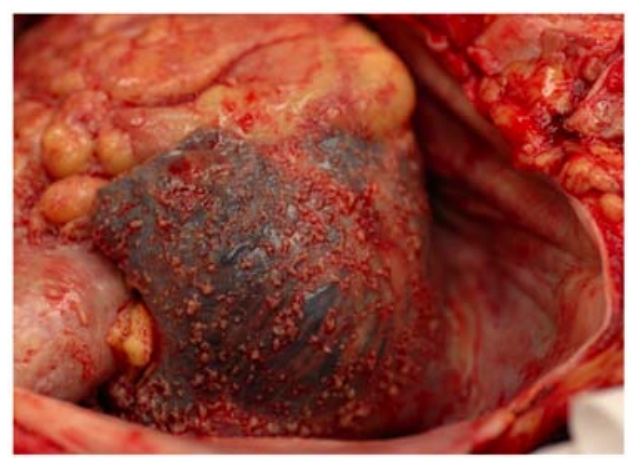

Figure 8. Macroscopic autopsy observations. Bleeding in the pericardial space resulted in cardiac tamponade. Diffuse and evident pericardial dissemination was observed.

were performed on $3 \mu \mathrm{m}$-thick sections of paraffin embedded specimen, fixed in $10 \%$ formalin at room temperature for $24 \mathrm{~h}$, and stained with $0.1 \%$ hematoxylin for $4 \mathrm{~min}$ and $1 \%$ eosin for 2 min. Diffuse lymphangitic carcinomatosis of the left lung 
A

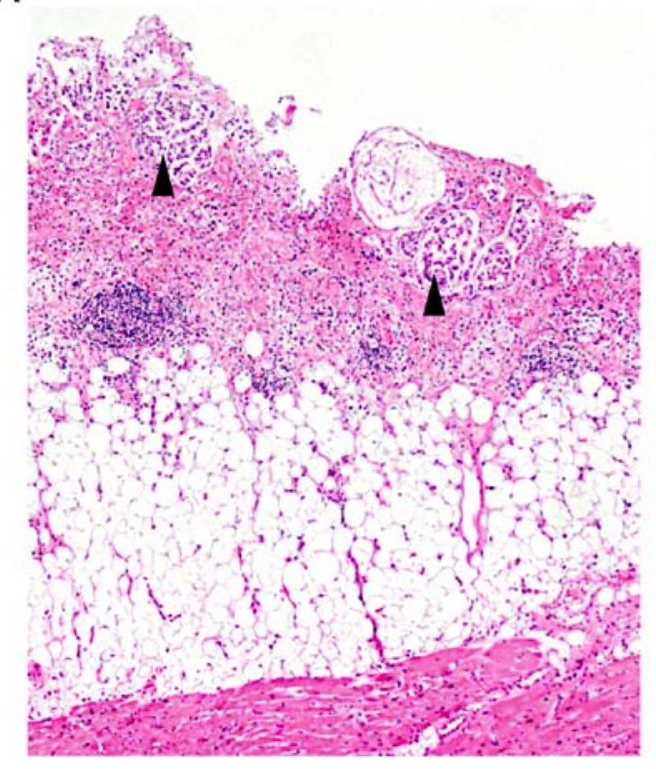

B

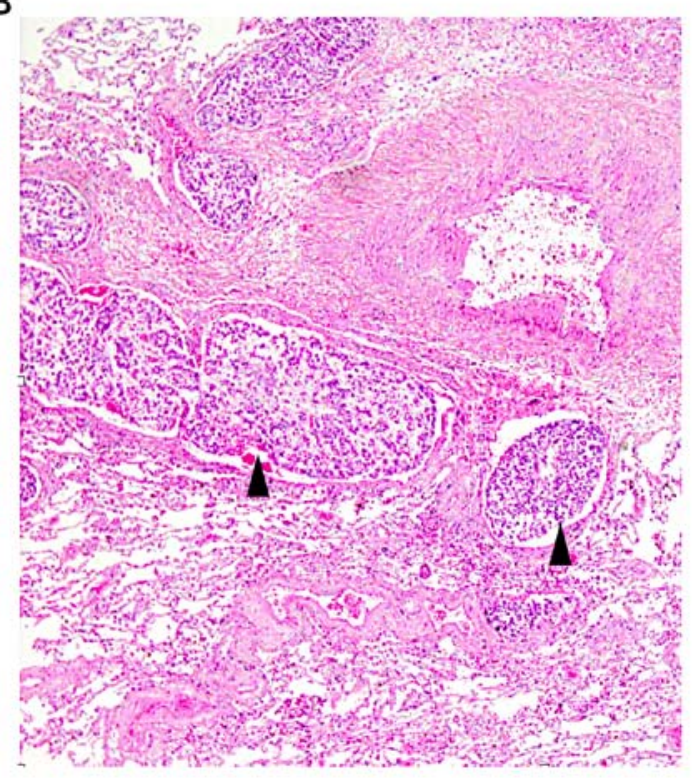

Figure 9. Microscopic observations. Disseminated viable cancer cells were found in the dilated lymphatic vessels of (A) the pericardium (hematoxylin and eosin staining; magnification, x100) and (B) the left lung (hematoxylin and eosin staining; magnification, x200). Arrows indicate tumor cells in the dilated lymphatic vessels.

without macroscopic nodules and moderately differentiated adenocarcinoma were observed throughout both lungs and the pericardium, with evidence of lymphatic invasion without tumor necrosis (Fig. 9). The histological findings thus indicate that chemotherapy has minimal effect.

\section{Discussion}

Regorafenib markedly extend the progression-free survival time (PFS) and overall survival time (OS) in patients with pretreated metastatic colorectal carcinoma (mCRC) in the international phase III CORRECT study (NCT 01103323) (1-4). Dose modification, due to toxicity associated with treatment, was required in $49 \%$ of cases in the CONSIGN Phase IIIb study (7). Additionally, recent data on the real clinical practice of using regorafenib for treatment have been collated, which revealed that treatment was initiated at a $25 \%$-decreased dose (120 mg/day) in 59- and 50\% -decreased dose (80 mg/day) in $13 \%$ of cases, in order to avoid adverse effects due to toxicity (such as hand-foot syndrome or general fatigue) (8). Bekaii-Saab et al (9) reported that the dose-escalation strategy starting from half of the regulated starting dose (160 mg once daily) provides a safe and effective alternative with improved of quality of life of the patients during treatment in phase II study.

Previous studies have reported observation of a radiological response as defined by the response evaluation criteria in solid tumors criteria (version 1.1) (10) in $1-4.4 \%$ of patients $(1,2,11)$. Minimal to no tumor growth $(\leq 9 \%$ increase in the sum of target-lesion diameters) in the 8th week of treatment was considered a predictive marker of improvement in the OS as defined by the RadioCORRECT post-hoc radiological analysis (12); however, this series did not include cases of partial response or complete response, and the disease control rate was $53.4 \%$ (12). In some studies, decreased density of liver metastases and cavitating pulmonary metastases, which are characteristic radiological changes induced by antiangiogenic agents, are reported as predictive markers of improved prognosis $(13,14)$. The RadioCORRECT study reported that these radiological findings do not affect the OS or PFS. Furthermore, Lim et al (15) reported that the magnitude of change in the tumor density does not affect the clinical outcome.

Other agents, such as TAS102, have also proven to exert long-lasting effects on the OS of patients with mCRC where salvage treatment has not resulted in improvement of overall response rate $(3,4)$.

Lymphangitic carcinomatosis is essentially the infiltration of the lung lymphatics with malignant cancer cells; symptoms manifest as an increase in lung tissue permeability (16-18). Previous studies involving radiological imaging have shown that early reduction of tumor perfusion and vascularity can be achieved by administration of regorafenib in a xenograft model $(19,20)$. Furthermore, lymphatic spread of tumor cells is accelerated by lymphangiogenesis promoted by VEGF-C and VEGF-D, which bind to the VEGF receptor-3 on lymphatic endothelial cells $(21,22)$. This receptor is a target of regorafenib, which acts as a multitarget tyrosine kinase inhibitor (19).

In the present case, regorafenib induced quick and prominent reduction in lung permeability resulting in early improvement of respiratory distress. This could be attributed to the effect of regorafenib on tissue permeability, as established in previous xenograft model studies $(19,20)$.

Histological examination of the left lung reveled viable tumor cells in the dilated lymphatic vessels. In contrast, clinical imaging did not show lymphangitic carcinomatosis until the death of the patient owing to pericardial dissemination. No evident change in the increased levels of carcinoembryonic antigen (above the normal range) during treatment suggested that regorafenib had markedly improved lymphangitis and maintained the peritumoral osmotic pressure, with a moderate 
effect on lymphatic invasion and minimal direct effect on cancer cells. These results might be attributed to the effects of regorafenib on the VEGF receptor-1, -2 , and -3 .

In conclusion, the present study reports a case in which regorafenib markedly reduced peritumoral edema via its multityrosine kinase inhibitor activity toward VEGF receptors. The administration of regorafenib rapidly alleviated the symptoms of lymphangitic carcinomatosis, despite its inadequate potency.

The treatment regimen for regorafenib administration requires further improvement to increase safety and efficacy $(9,23)$. In addition, combination treatment of regorafenib with other cytotoxic agent warrants further investigation (24); however, this represents a promising approach for the treatment of osmotic abnormalities in the peritumoral environment.

\section{Acknowledgements}

Not applicable.

\section{Funding}

No funding received.

\section{Availability of data and materials}

The data used and/or analyzed during the current study are available from the corresponding author on reasonable request.

\section{Authors' contributions}

NT collected and analyzed clinical data and literature, and drafted, designed, wrote and revised the manuscript. KK collected clinical data during treatment, designed figures and drafted the manuscript. SY collected clinical data of previous treatment in another hospital and drafted the manuscript. MF reviewed the pathological findings and revised the manuscript. All authors have approved the manuscript and agreed with its submission.

\section{Ethics approval and consent to participate}

Not applicable.

\section{Patient consent for publication}

Written informed consent was obtained from the patient's sister for publication of this article and the accompanying images.

\section{Competing interests}

The authors declare that they have no competing interests.

\section{References}

1. Grothey A, Van Cutsem E, Sobrero A, Siena S, Falcone A, Ychou M Humblet Y, Bouché O, Mineur L, Barone C, et al: Regorafenib monotherapy for previously treated metastatic colorectal cancer (CORRECT): An international, multicentre, randomised, placebo-controlled, phase 3 trial. Lancet 381: 303-312, 2013.
2. Li J, Qin S, Xu R, Yau TC, Ma B, Pan H, Xu J, Bai Y, Chi Y, Wang L, et al: Regorafenib plus best supportive care versus placebo plus best supportive care in Asian patients with previously treated metastatic colorectal cancer (CONCUR): A randomised, double-blind, placebo-controlled, phase 3 trial. Lancet Oncol 16: 619-629, 2015.

3. Mayer RJ, Van Cutsem E, Falcone A, Yoshino T, Garcia-Carbonero R, Mizunuma N, Yamazaki K, Shimada Y Tabernero J, Komatsu Y, et al: Randomized trial of TAS-102 for refractory metastatic colorectal cancer. N Engl J Med 372: 1909-1919, 2015

4. Xu J, Kim TW, Shen L, Sriuranpong V, Pan H, Xu R, Guo W, Han SW, Liu T, Park YS, et al: Results of a randomized, double-blind, placebo-controlled, Phase III trial of trifluridine/tipiracil (TAS-102) monotherapy in Asian patients with previously treated metastatic colorectal cancer: The TERRA study. J Clin Oncol 36: 350-358, 2018.

5. National Collaborating Centre for Chronic Conditions: Chronic obstructive pulmonary disease: National clinical guideline for management of chronic obstructive pulmonary disease in adults in primary and secondary care. Thorax 59 (Suppl 1): S1-S232, 2004.

6. Andrassy KM: Comments on KDIGO 2012 clinical practice guideline for the evaluation and management of chronic kidney disease. Kidney Int 84: 622-623, 2013.

7. Van Cutsem E, Martinelli E, Cascinu S, Sobrero A, Banzi M, Seitz JF, Barone C, Ychou M, Peeters M, Brenner B, et al: Regorafenib for patients with metastatic colorectal cancer who progressed after standard therapy: Results of the large, single-arm, open-label phase IIIb CONSIGN study. Oncologist 24: 185-192, 2019.

8. Ramaswamy A, Ostwal V, Pande N, Sharma A, Patil S, Thippeswamy R, Ghadyalpatil N, Roy R, Peshwe H, Poladia B, et al: Practice patterns and outcomes with the use of regorafenib in metastatic colorectal cancer: Results from the Regorafenib in metastatic colorectal cancer-an Indian Exploratory analysis study. South Asian J Cancer 8: 22-26, 2019.

9. Bekaii-Saab TS, Ou FS, Ahn DH, Boland PM, Ciombor KK, Heying EN, Dockter TJ, Jacobs NL, Pasche BC, Cleary JM, et al: Regorafenib dose-optimisation in patients with refractory metastatic colorectal cancer (ReDOS): A randomised, multicentre, open-label, phase 2 study. Lancet Oncol 20: 1070-1082, 2019.

10. Eisenhauer EA, Therasse P, Bogaerts J, Schwartz LH, Sargent D, Ford R, Dancey J, Arbuck S, Gwyther S, Mooney M, et al: New reponse evaluation criteria in solid tumours: Revised RECIST guideline (version 1.1) Euro J Cancer 45: 228-247, 2009.

11. Yoshino T, Komatsu Y, Yamada Y, Yamazaki K, Tsuji A, Ura T, Grothey A, Van Cutsem E, Wagner A, Cihon F, et al: Randomized phase III trial of regorafenib in metastatic colorectal cancer: Analysis of the CORRECT Japanese and non-Japanese subpopulations. Invest New Drugs 33: 740-750, 2015.

12. Ricotta R, Verrioli A, Ghezzi S, Porcu L, Grothey A, Falcone A, Van Cutsem E, Argilés G, Adenis A, Ychou M, et al: Radiological imaging markers predicting clinical outcome in patients with metastatic colorectal carcinoma treated with regorafenib: Post hoc analysis of the CORRECT phase III trial (RadioCORRECT study). ESMO Open 1: e000111, 2017.

13. Nishino M, Cryer SK, Okajima Y, Sholl LM, Hatabu H, Rabin MS, Jackman DM and Johnson BE: Tumoral cavitation in patients with non-small-cell lung cancer treated with antiangiogenic therapy using bevacizumab. Cancer Imaging 12: 225-235, 2012.

14. Boonsirikamchai P, Asran MA, Maru DM, Vauthey JN, Kaur H, Kopetz S and Loyer EM: CT findings of response and recurrence, independent of change in tumor size, in colorectal liver metastasis treated with bevacizumab. AJR Am J Roentgenol 197: W1060-W1066, 2011.

15. Lim Y, Han SW, Yoon JH, Lee JM, Lee JM, Paeng JC, Won JK, Kang GH, Jeong SY, Park KJ, et al: Clinical implication of anti-angiogenic effect of regorafenib in metastatic colorectal cancer. PLoS One 10: e0145004, 2015.

16. Chandler GN and Telling M: Lymphangitis carcinomatosa $\mathrm{Br}$ Med J 2: 639-641, 1952

17. Thomas A and Lenox R: Pulmonary lymphangitic carcinomatosis as a primary manifestation of colon cancer in a young adult. CMAJ 179: 338-340, 2008.

18. Bruce DM, Heys SD and Eremin O: Lymphangitis carcinomatosa: A literature review. J R Coll Surg Edinb 41: 7-13, 1996. 
19. Cyran CC, Kazmierczak PM, Hirner H, Moser M, Ingrisch M, Havla L, Michels A, Eschbach R, Schwarz B, Reiser MF, et al: Regorafenib effects on human colon carcinoma xenografts monitored by dynamic contrast-enhanced computed tomography with immunohistochemical validation. PLoS One 8: e76009, 2013

20. Wilhelm SM, Dumas J, Adnane L,Lynch M, Carter CA, Schütz G Thierauch KH and Zopf D: Regorafenib (BAY 73-4506): A new oral multikinase inhibitor of angiogenic, stromal and oncogenic receptor tyrosine kinases with potent preclinical antitumor activity. Int J Cancer 129: 245-255, 2011.

21. He Y, Rajantie I, Pajusola K, Jeltsch M, Holopainen T, Yla-Herttuala S, Harding T, Jooss K, Takahashi T and Alitalo K: Vascular endothelial cell growth factor receptor 3-mediated activation of lymphatic endothelium is crucial for tumor cell entry and spread via lymphatic vessels. Cancer Res 65: 4739-4746, 2005

22. Shimizu K, Kubo H, Yamaguchi K, Kawashima K, Ueda Y, Matsuo K, Awane M, Shimahara Y, Takabayashi A, Yamaoka Y and Satoh S: Suppression of VEGFR-3 signaling inhibits lymph node metastasis in gastric cancer. Cancer Sci 95: 328-333, 2004
23. Bekaii-Saab T, Kim R, Kim TW, O'Connor JM, Strickler JH, Malka D, Sartore-Bianchi A, Bi F, Yamaguchi K, Yoshino T and Prager GW: Third- or later-line therapy for metastatic colorectal cancer: Reviewing best practice. Clin Colorectal Cancer 18: e117-e129, 2019.

24. Schultheis B, Folprecht G, Kuhlmann J, Ehrenberg R, Hacker UT, Köhne CH, Kornacker M, Boix O, Lettieri J, Krauss J, et al: Regorafenib in combination with FOLFOX or FOLFIRI as first- or second-line treatment of colorectal cancer: Results of a multicenter, phase Ib study. Ann Oncol 24: 1560-1567, 2013.

This work is licensed under a Creative Commons Attribution-NonCommercial-NoDerivatives 4.0 International (CC BY-NC-ND 4.0) License. 